Published in final edited form as:

Nat Microbiol. 2018 June ; 3(6): 687-697. doi:10.1038/s41564-018-0162-2.

\title{
Genomes of all known members of a Plasmodium subgenus reveal paths to virulent human malaria
}

\author{
Thomas D. Otto ${ }^{1, \dagger, \#,{ }^{*}, \text { Aude Gilabert }}{ }^{2, \dagger}$, Thomas Crellen $^{1,3}$, Ulrike Böhme $^{1}$, Céline \\ Arnathau $^{2}$, Mandy Sanders ${ }^{1}$, Samuel O. Oyola ${ }^{1,4}$, Alain Prince Okouga ${ }^{5}$, Larson \\ Boundenga $^{5}$, Eric Willaume ${ }^{6}$, Barthélémy Ngoubangoye ${ }^{5}$, Nancy Diamella Moukodoum ${ }^{5}$, \\ Christophe Paupy ${ }^{2}$, Patrick Durand ${ }^{2}$, Virginie Rougeron ${ }^{2,5}$, Benjamin Ollomo ${ }^{5}$, François \\ Renaud $^{2}$, Chris Newbold ${ }^{1,7}$, Matthew Berriman ${ }^{1,{ }^{*}}$, and Franck Prugnolle ${ }^{2,5,{ }^{*}}$ \\ ${ }^{1}$ Wellcome Trust Sanger Institute, Wellcome Genome Campus, Hinxton CB10 1SA, United \\ Kingdom \\ 2Laboratoire MIVEGEC, UMR 5290-224 CNRS 5290-IRD 224-UM, Montpellier, France \\ ${ }^{3}$ Department of Infectious Disease Epidemiology, Imperial College London, St Mary's Campus, \\ Norfolk Place, London W2 1PG, United Kingdom \\ ${ }^{5}$ Centre International de Recherches Médicales de Franceville, Franceville, Gabon \\ ${ }^{6}$ Sodepal, Parc of la Lékédi, Bakoumba, Gabon \\ ${ }^{7}$ Weatherall Institute of Molecular Medicine, University of Oxford, John Radcliffe Hospital, Oxford \\ OX3 9DS, United Kingdom
}

\begin{abstract}
Plasmodium falciparum, the most virulent agent of human malaria, shares a recent common ancestor with the gorilla parasite $P$. praefalciparum. Little is known about the other gorilla and chimpanzee-infecting species in the same (Laverania) subgenus as $P$. falciparum but none of them
\end{abstract}

\footnotetext{
Users may view, print, copy, and download text and data-mine the content in such documents, for the purposes of academic research, subject always to the full Conditions of use:http://www.nature.com/authors/editorial_policies/license.html\#terms

"Correspondence to: Thomas D. Otto (thomasdan.otto@glasgow.ac.uk), Matthew Berriman (mb4@ sanger.ac.uk) or Franck Prugnolle (franck.prugnolle@ird.fr).

4 International Livestock Research Institute, Box 30709, Nairobi, Kenya (current address)

\#Current Address: Centre of Immunobiology, Institute of Infection, Immunity \& Inflammation, College of Medical, Veterinary and

Life Sciences, University of Glasgow, Glasgow, United Kingdom

$\dagger$ These authors contributed equally.

Author Contributions: TDO, BO, FR, CN, MB, FP designed the study. CA, APO, LB, EW, BN, ND, CP, PD, VR, FP collected and assessed samples. CA performed the WGA and cell sorting on one sample. SO performed the WGA on the samples; MS organised the sequencing. TDO did assembly and annotation. UB did manual gene curation; AG, FP performed the evolutionary analyses on core genomes. TDO, CN, MB performed the analyses of gene families and dimorphisms. TC performed the dating analyses. TDO, AG, $\mathrm{CN}, \mathrm{MB}, \mathrm{FP}$ wrote the manuscript. All authors read and approved the paper.

Data availability

All sequences have been submitted to the European Nucleotide Archive. The accession numbers of the raw reads, and assembly data can be found in Supplementary Table 9. The genomes are being submitted to EBI, project ID PRJEB13584. The genomes are available from plasmodb.org and from ftp://ftp.sanger.ac.uk/pub/project/pathogens/Plasmodium/Laverania/.

Competing financial interests.

None

Computer code

Custom computer code is available on request.
} 
are capable of establishing repeated infection and transmission in humans. To elucidate underlying mechanisms and the evolutionary history of this subgenus, we have generated multiple genomes from all known Laverania species. The completeness of our dataset allows us to conclude that interspecific gene transfers as well as convergent evolution were important in the evolution of these species. Striking copy number and structural variations were observed within gene families and one, stevor shows a host specific sequence pattern. The complete genome sequence of the closest ancestor of $P$. falciparum enables us to estimate the timing of the beginning of speciation to be 40,000-60,000 years ago followed by a population bottleneck around 4,000-6,000 years ago. Our data allow us also to search in detail for the features of $P$. falciparum that made it the only member of the Laverania able to infect and spread in humans.

The evolutionary history of Plasmodium falciparum, the most common and deadly human malaria parasite, has been the subject of uncertainty and debate1,2. Recently it has become clear that $P$. falciparum is derived from a group of parasites infecting African Great Apes, known as the Laverania subgenus2. Until 2009, chimpanzee-infecting $P$. reichenowi was the only other species known in this subgenus, for which only one isolate was available3. It is now clear that there are a total of at least seven species in Great Apes that naturally infect chimpanzees ( $P$. gaboni, $P$. billcollinsi and $P$. reichenowi), gorillas (P. praefalciparum, $P$. blacklocki and $P$. adleri) 4,5, or humans ( $P$. falciparum) (Fig. 1a). Within this group, $P$. falciparum is the only parasite that has successfully adapted to humans after a transfer from gorillas and subsequently spread worldwide2.

Over time there have been various estimates concerning the evolutionary history of $P$. falciparum with the speciation event having been estimated to be anywhere between 10,000 to 5.5 million years ago, the latter falsely based on the date of the chimpanzee-human split6,7. Others report a bottleneck less than 10,000 years ago8, but suggest a drop to a single progenitor parasite. The latter seems unlikely due to the presence of allelic dimorphisms that predate speciation events and could not have both been transmitted if a new species were founded by a single individual infection. Also, the dating of the speciation cannot be accurately estimated without the genome sequence of $P$. praefalciparum, the closest living sister species to $P$. falciparum.

The absence of in vitro culture or an animal model has precluded obtaining sufficient DNA for full genome sequencing and has hindered investigation of the Laverania. So far the full draft genome of $P$. reichenow $P$ and a nearly complete draft sequence of $P$. gaboni6 are available. These data together with additional PCR based approaches 10 have provided important insights into the evolution of this subgenus, including the lateral gene transfer of the $r h 5$ locus, the early expansion of the FIKK gene family and the observation that the common ancestor also had vargenes. Our data confirm and significantly extend these findings. However, the lack of whole genome information for the whole subgenus (particularly P. praefalciparum) has severely constrained the scope of subsequent analyses.

To investigate the evolutionary history of all known members of the Laverania subgenus and to address the question of why $P$. falciparum is the only extant species to have adapted successfully to humans, we have sequenced multiple genotypes of all known Laverania species. 


\section{Genome sequencing from six Laverania species}

Fifteen blood samples that were positive for ape malaria parasites by PCR were taken during successive routine sanitary controls, from four gorillas and seven chimpanzees living in a sanctuary or quarantine facility prior to release (see Methods). Despite low parasitemia, a combination of host DNA depletion, parasite cell sorting and amplification methods enabled sufficient parasite DNA templates to be obtained for short- (Illumina) and long- read (Pacific Bioscience) sequencing (Supplementary Table 1). Mixed-species infections were frequent but resolved by utilising sequence data from single infections, resulting in 19 genotypes (Supplementary Table 1). The dominant genotype in each sample was assembled de novo (see Methods) using long read technology into a reference genome for six malaria parasite species: P. praefalciparum, P. blacklocki, P. adleri, P. billcollinsi, P. gaboni and P. reichenowi. The assemblies comprised 44-97 scaffolds (Supplementary Table 1), with large contigs containing the subtelomeric regions and internal gene clusters that house multigene families known in P. falciparum and P. reichenowi to be involved in virulence and host-pathogen interactions. The high quality of the assemblies compared to those obtained previously is illustrated by the good representation of multi-gene families (Supplementary Table 2) and the large number of one-to-one orthologues obtained between the different reference genomes ( 4,350 among the seven species and 4,826 between $P$. falciparum, $P$. praefalciparum and $P$. reichenowi). Two to four additional genomes were obtained for each species except for $P$. blacklocki (Supplementary Table 1).

\section{Speciation history in the Laverania sub-genus}

Conservation of synteny is striking between these complete genomes and enabled us to reconstruct the relationships between different Laverania species, to compare their relative genetic diversity (Fig 2a, Supplementary Fig. 1) and to estimate the age of the different speciation events that led to the extant species. The latter has been problematic in the past due to the lack of both complete genome data and accurate estimates of mutation rate and generation time. Using the most divergent estimates of generation time and measured mutation rates from the $P$. falciparum literature, we found the data converge to 0.9-1.5 mutations per year per genome (Supplementary Note 1). We observed a similar substitution rate in vivo by examining existing sequence data for five geographically diverse isolates, covering a 200-kb region surrounding the PfCRT gene that is relatively conserved due to a selective sweep resulting from chloroquine use (Supplementary Note 1; Supplementary Figure 2). The fact that these two figures are similar suggests that the in vitro mutation rate may have been underestimated since many mutations will be lost by genetic drift. Since no data is available for the other species we have assumed hereon that these values generalise across the subgenus. From Bayesian whole-genome estimates, the ancestor of all current day parasites of this subgenus existed 0.7-1.2 million years ago, a time at which the subgenus divided into two main clades, A ( $P$. adleri and $P$. gaboni) and B that includes the remaining species (Fig. 1a). Our range of values is far more recent than previous estimates3,11. Following the Clade A/B subdivision, several speciation events occurred leading either to new chimpanzee or gorilla parasites. Interestingly, the divergence between $P$. adleri and $P$. gaboni in one lineage and $P$. reichenowi and the ancestor of $P$. praefalciparum/P. falciparum in the other lineage occurred at approximately the same time (140-230 thousand years ago; 
Fig. 1a, Table 2). Based on coalescence estimates, $P$. falciparum begun to emerge in humans from $P$. praefalciparum around 40-60 thousand years ago (Fig. 1a), significantly later than the evolution of the first modern humans and their spread throughout Africa12. Our analysis also indicates significant gene flow between these two parasite species after their initial divergence (Supplementary Table 3).

P. falciparum has strikingly low diversity ( $\pi=0.0004)$, compared with the other Laverania species (0.002-0.0049) (Supplementary Fig. 1). It has been proposed that $P$. falciparum arose from a single transfer of $P$. praefalciparum into humans6 and based in part on the paucity of neutral SNPs within the genome, that $P$. falciparum emerged from a bottleneck of a single parasite around 10,000 years ago, after agriculture was established6,8. In light of our results, we estimate that the $P$. falciparum population declined around 11,000 years ago and reached a minimum about 5,000 years ago (Fig. 1b) with an effective population size $(\mathrm{Ne}$ ) of around 3,000 (Supplementary Note 1; generally the census number of parasites is higher than Ne13). The hypothesis of a single progenitor is also inconsistent with the observation of several ancient gene dimorphisms that have been observed in P. falciparum. A previous analysis using $P$. reichenowi and $P$. gaboni sequence data, provided some evidence that different dimorphic loci diverged at different points in the tree14. Looking at each of these $P$. falciparum loci across the Laverania, we found different patterns of evolution at the msp1, var1csa, and msp3 loci (Supplementary Fig. 3a). Most strikingly, a mutually exclusive dimorphism (described as MAD20/K115) in the central 70\% of the msp1 sequence, clearly pre-dates the $P$. falciparum-P. praefalciparum common ancestor and dimorphism in varlcsa (an unusual var gene of unknown function that is transcribed late in the asexual cycle) occurred before the split with $P$. reichenowi.

In contrast, the gene eba-175 that encodes a parasite surface ligand involved in red blood cell invasion contains a dimorphism that arose after the emergence of $P$. falciparum

(Supplementary Fig. 3b). The time to the most recent common ancestor of eba-175 has been estimated as 130-140 thousand years in an analysis 16 that assumed $P$. falciparum and $P$. reichenowi diverged 6 million years ago. However, based on our new estimate for $P$. falciparum- $P$. reichenowi divergence, we recalibrated their estimate of the most recent common ancestor of the eba-175 alleles to be around 4,000 years ago, which is in good agreement with our divergence time for $P$. falciparum (Supplementary Note 1). The recent dimorphism cannot however explain the observation of an ancient dimorphism near the human and ape loci for glycophorin17 - an EBA-175 binding protein. The formation and maintenance of all of these dimorphic loci has therefore been shaped by different balancing selection pressures over time.

\section{P. falciparum-specific evolution}

During its move away from gorillas, $P$. falciparum had to adapt to a new vertebrate host (human) and new vector species (e.g. Anopheles gambiae)18. To infer $P$. falciparum specific adaptive changes, we considered the $P$. falciparum / P. praefalciparum /P. reichenowi genome trio and then applied two lineage based tests to find positive selection that occurred in the $P$. falciparum branch (see methods). The two tests identified 172 genes (out of 4,826) with signatures of positive selection in the human parasite species only (Supplementary Table 4). 
Two genes (rop14 and PF3D7_0609900) were significant in both tests. Among the 172 genes, almost half $(n=82)$ encoded proteins of unknown function. Analysis of those with functional annotation indicated that genes involved in pathogenesis, entry into host, actin movement and organization and in drug response were significantly over-represented. Other genes, expressed in different stages of the P. falciparum life cycle (e.g. sera4 and emp3, involved during the erythrocytic stages; trsp and lisp1, involved in the hepatic stages; and plp4, CelTOS and Cap380, involved in the mosquito stages) also showed a significant signal of adaptive evolution (Supplementary Table 4).

\section{Evolution through introgression, gene transfer and convergence}

Frequent mixed species infections in apes and mosquitoes 18 provide clear opportunities for interspecific gene flow between these parasites. A recent study6 reported a gene transfer event between $P$. adleri and the ancestor of $P$. falciparum and $P$. praefalciparum of a region on chromosome 4 including key genes involved in erythrocyte invasion ( $r h r 5$ and cyrpA). Because such events preserve the phylogenetic history of the genes involved, we systematically examined the evidence for introgression or gene transfer events across the complete subgenus by testing the congruence of each gene tree to the species tree for genes with one-to-one orthologues. Beyond the region that includes rh5 (Fig 2b, Supplementary Fig. 4a), few signals of gene flow between parasites infecting the same host species were obtained ( $\mathrm{n}=11$ ) suggesting that these events were rare or usually strongly deleterious (Supplementary Fig. 5).

The Laverania subgenus evolved to infect chimpanzees and gorillas but, on a genome-wide scale, the convergent evolution of host-specific traits has not left a signature (Supplementary Note 2). We therefore examined each CDS independently and were able to identify genes with differences fixed within specific hosts, falling into three categories: 53 in chimpanzeeinfective parasites, 49 in gorilla-infective and 12 with fixed traits in both host species (Fig. 2; Supplementary Table 5a). For at least 67 genes, these differences were unlikely to have arisen by chance $(\mathrm{p}<0.05)$ and GO term enrichment analysis revealed that several of these genes are involved in host invasion and pathogenesis (Supplementary Table 5b) including rh5 (which has a signal for convergent evolution even when the introgressed tree topology is taken into consideration; Supplementary Fig. 4b). Rh5 is the only gene identified in $P$. falciparum that is essential for erythrocyte recognition during invasion, via binding to Basigin. P. falciparum rh 5 cannot bind to gorilla Basigin and binds poorly to the chimpanzee protein19. We notice that one of the convergent sites is known to be a binding site for the host receptor Basigin20 (Supplementary Fig. 4b). The gene eba-165 encodes a member of the erythrocyte binding like (EBL) super family of proteins that are involved in erythrocyte invasion. Although eba-165 is a pseudogene in P. falciparum21, it is not a pseudogene in the other Laverania species and may therefore be involved in erythrocyte invasion, like other EBL members. The protein has three convergent sites in gorillas. One falls inside the F2 region, a domain involved in the interactions with erythrocyte receptors. The role of this protein and of these convergent sites in the invasion of gorilla red cells remains to be determined. Finally, genes involved in gamete fertility (the 6-cysteine protein P230) or implicated in Plasmodium invasion of erythrocytes (doc222) also displayed signals of convergent evolution. Twelve parasite coding sequences had fixed differences at the same 
amino-acid position in chimpanzees and gorillas. Of these P230 was the only one found with a position that was different and fixed across all three host species. P230 is involved in gamete development and trans-specific reproductive barriers 23 , possibly through enabling male gametes to bind to erythrocytes prior to exflagellation24. Host-specific residues observed in P230 might affect the efficiency of the binding to the erythrocyte receptors and result from co-evolution between the parasite molecule and the host receptor.

\section{Subtelomeric gene families}

To date, the only in-depth data on the subtelomeric gene families of the Laverania have come from $P$. reichenowi and $P$. falciparum. These important families are well represented in our assemblies (Supplementary Tables 2 and 6A) and we provide a comprehensive picture of their evolution.

Most gene families were likely present in the ancestor of all Laverania. The same general pattern of one-to-one orthology throughout the subgenus indicates that many underwent gene duplication early (e.g. FIKK) or prior (e.g. ETRAMP, PHIST and SURFIN) to the development of a distinct Laverania lineage. Only a subset displayed contractions or expansions between specific Laverania species (Fig. 3 and Supplementary Table 6a, Supplementary Figure 7). For these latter families, Clade A and most species of Clade B clearly differ in their composition. P. blacklocki (Clade B) is intermediate in its composition. Some gene families, like the group of exported proteins hyp4, hyp5, mc-2tm and EPF1, have expanded only in $P$. praefalciparum and $P$. falciparum (and even more in $P$. falciparum for hyp4 and hyp5). Since all four are components of Maurer's clefts, an organelle involved in protein export25, some evolution of function in this organelle may have been an important precursor to human infection. The family of acyl-CoA synthetase genes, reported to be expanded and diversified in $P$. falciparum 26 is in fact expanded across the Laverania and has four fewer copies in $P$. falciparum (Supplementary Fig. 6). Other genes that show clade or group specific expansion include DBLmsp, glycophorin binding protein and CLAG (Supplementary Fig. 7).

One striking inter-clade difference concerns the largest gene family that is likely common to all other malaria species: the Plasmodium interspersed repeat family (pir, which includes the rif and stevor families in P. falciparum) (Fig. 3, 4). This family has been proposed to be involved in important functions such as antigenic variation, immune evasion, signalling, trafficking, red cell rigidity and adhesion 27 and yet has expanded only in Clade B, after the P. blacklocki split (Fig 3). The rif genes comprise a small conserved group and a much larger group of more diverse members that contains just 13 genes from Clade A species and at least 180 members per Clade B species (Fig. 4). There is however no evidence for host-specific adaptation in these sequences.

In contrast, a subset of stevor genes showed strong host-specific sequence diversification (Fig 4 and Supplementary Fig. 8). Based on full-length alignments, there is a deep phylogenetic split between stevor genes but when only short conserved protein motifs are considered, a group of Stevor proteins (stevor II, Fig 4a) forms a cluster comprising almost entirely of members from gorilla-infecting species. Since stevor genes are known to be 
involved in host-parasite interactions (such as binding to host glycophorin $\mathrm{C}$ in $P$.

falciparum28), this host specific sequence may reflect sequence differences in host-specific factors in gorillas.

\section{Evolution of var genes}

The vargenes, crucial mediators of pathogenesis and the establishment of chronic infection through cytoadherence and immune evasion, are the best studied $P$. falciparum multi-gene family and unique to the Laverania29. They are two-exon genes and their products have three types of major domain; exon 1 encodes Duffy Binding like (DBL) and Cysteine Rich Interdomain Regions (CIDR) and exon 2 encodes Acidic Terminal Sequence (ATS)30. Similar to $P$. falciparum, our data are consistent with all Laverania species having var genes (Fig. 3) that retain a two-exon structure and are organized into subtelomeric or internal var gene clusters. There are however three notable features of var evolution within the subgenus.

First, there is a deep division in how the repertoire is organised between the major clades. The vargenes of Clade B parasites, with the exception of $P$. blacklocki, resemble those of $P$. falciparum in terms of genomic organisation, domain types and numbers (Fig 5, Supplementary Table 7). In contrast, the repertoires of Clade A parasites and P. blacklocki (treated as one group hereafter in this section) differ in their domain composition, contain a novel CIDR-like domain (CIDRn, Fig 5a, Supplementary Fig. 9) and have lower sequence diversity per domain but cluster into more sub-groups than Clade B domains (Fig 5b, Supplementary Fig. 10). The paucity of domains similar to those in P. falciparum (such as CIDRa) that are involved in cytoadherence to some specific and common host receptors, means that if endothelial cytoadherence was important in Clade A, some alternative receptors must have been utilised.

Second, in total there are 10 internal var gene clusters (confirmed by contiguous sequence data) but 8 are oppositely oriented between the two clades (Supplementary Fig. 11, Supplementary Table 8). Clade B parasites also show a much greater number of associated GC-rich RNAs of unknown Function (RUF) elements than Clade A (Supplementary Table $8)$.

Third, the ATS domains cluster tightly within Clade A. Within Clade B there is clear evidence of species-specific diversification, except in $P$. praefalciparum and $P$. falciparum reflecting their recent speciation. There is one intact ATS from $P$. falciparum as well as several pseudogenes that cluster with Clade A (Fig 5c). Moreover, of seven internal var arrays (Supplementary Fig. 11) in $P$. falciparum, containing a functional var gene, five terminate with one of these pseudogenes (on the opposite DNA strand) suggesting that they may be remnants from ancient rearrangements. The intact $P$. falciparum gene is var $2 c s a$, a var-like gene that is highly conserved between $P$. falciparum isolates 31 , involved both in cytoadherence in the placenta in primigravidae, and proposed to be a central intermediate in vargene switching during antigenic variation32. We therefore propose var2csa is a remnant of an ancient multigene family that has been maintained as a single complete gene in $P$. falciparum, for the dual purposes of var-switching and placental cytoadherence. 
There is other evidence of retention of ancient var gene sequence across the subgenus. First, in Clade B we find a nearly full length var pseudogene that has highest similarity to $P$. adleri and $P$. gaboni vargenes, within an internal var cluster on chromosome 4 in $P$. falciparum and $P$. praefalciparum but on the opposite strand to the other vargenes. It is found in all $P$. falciparum isolates, but not in $P$. reichenowi. Second, in $P$. gaboni and $P$. adleri, three genes have the N-terminal DBLa/CIDRa architecture typical of Clade B genes and their domains cluster within Clade B based on similarity (Fig. 5b, larger nodes). Directly adjacent to two of these vargenes are two rif pseudogenes that also show greatest similarity to those from Clade B. Last, we find a further nine rif pseudogenes of Clade A parasites that cluster with Clade B rif genes (Fig. 4). If these observations reflect retention of ancient copies, their high sequence conservation suggests that they are under extremely unusual selection pressure. Alternatively, they may represent relics of gene transfer between species that occurred after the Clade A/B split.

\section{Conclusion}

We have produced high quality genomes and used mutation rates and generation times, covering the full range of most recent estimates, to calculate the date of speciation for all known members of the Laverania, with only a small margin of error. In our analysis, we have shown that the successful infection of humans by $P$. falciparum occurred quite recently and involved numerous parasites rather than a single one as previously proposed. After the establishment in its new host, the parasite population went through a bottleneck around 5,000 years ago during the period of rapid human population expansion due to farming (Fig. 1b). We summarise the major genomic events during the evolution of the Laverania in Fig. 6.

As a result of our analyses we propose the following series of events for the emergence of $P$. falciparum as a major human pathogen. First, the crucial lateral transfer event of the rhs locus between Clade A and B parasites may also have involved var and rifin genes in other parts of the genome that, because of their orientation on the opposite strand, were not lost during later recombination. Next, facilitatory mutations are likely to have occurred in rh5 that in the first instance allowed invasion of both gorilla and human red cells. Modern humans emerged more than 300,000 years ago33 and existed as small isolated populations12. Our evidence suggests that $P$. falciparum and $P$. praefalciparum started to diverge around 40,000-60,000 years ago. In the following 40,000 years with low population densities in humans and gorillas there would have not been high selection pressure to optimise infectivity in either the hosts or vectors, enabling at least some movement of parasites between hosts. We find evidence for gene flow between lineages throughout this period. The expansion of the human population with the advent of farming likely led to strong evolutionary pressure for mosquito species (specifically An. gambiae) to feed primarily on humans34. Therefore, the existing human infective ( $P$. falciparum) genotypes would be selected for human and appropriate vector success and the fittest would rapidly expand. Subsequent rapid accumulation of mutations that favoured growth in humans, and in the anthropophilic vectors such as An. gambiae, are likely to have occurred to increase human-specific reproductive success. The resulting specific parasite genotypes that expanded (and appeared as an emergence from a bottleneck), would have had a much lower probability of a direct transfer back to apes. With experiments on gorillas and chimpanzees 
not possible it will be difficult directly to prove the precise combination of different alleles that allowed the emergence of $P$. falciparum. However, for the genes that we have implicated in this process, existing data (www.genedb.org, plasmodb.org) suggest they are expressed throughout the life cycle but that only half have been characterised. This opens up new opportunities for future studies on host specificity and host adaptation in Plasmodium.

\section{Online Methods}

\section{Sample collection}

All but two infected blood samples from chimpanzees (Pan troglodytes troglodytes) and gorillas (Gorilla gorilla gorilla) were obtained from the sanctuary "Parc de La Lékédi", Bakoumba (Haut-Ogooué, Gabon), during routine sanitary controls of the animals. This park holds various primate species, including gorillas, chimpanzees and monkeys (Cercopithecinae), that have been orphaned due to bushmeat-poaching activities and have been confiscated by the Gabonese Government, quarantined at the Centre International de Recherches Médicales de Franceville (CIRMF, Gabon) and finally released into semi-free ranging enclosures in the sanctuary. Every six months, chimpanzees (12 individuals) and gorillas ( 2 individuals) are anesthetized for medical check up. Blood samples were collected from the animals during sanitary controls (July 2011, September 2012, May 2013 and December 2013). Two additional infected blood samples were obtained from gorilla orphans (GG05, GG06) seized by the Gabonese government in 2011 and 2013 and sent to the CIRMF for a quarantine before being released in a sanctuary. All animal work was conducted according to relevant national and international guidelines. From each animal, 15 $\mathrm{ml}$ of whole blood were collected in EDTA tubes. For all samples but three, white blood cell depletion was performed on $10 \mathrm{ml}$ of the freshly collected samples using cellulose columns as described in 35. Remaining blood was subsequently used for DNA extraction and detection of Plasmodium infections as described in Ollomo et al3. Overall, 15 blood samples from 7 chimpanzees and 4 gorillas were found to contain the Laverania samples used in the present study (Supplementary Table 1).

\section{Ethical consideration}

The animal well-being was guaranteed by the veterinarians of the "Parc of la Lékédi" and the CIRMF who proceeded to the sanitary controls and the blood sampling. Because these blood samples were collected as part of the standard protocol for the sanitary controls (and not specifically for our experiment), our study did not need the approval of an Institutional Animal Care or Use Committee. Note also that our study did not involve randomisation nor blinding.

\section{Sample preparation}

Three methods were used for DNA amplification prior to sequencing (Supplementary Table 1). For all but one sample, whole genome amplification (WGA) was performed with a REPLI-g Mini Kit (Qiagen) following a modified protocol36 to enrich genomic DNA. The genome of $P$. blacklocki was generated using selective WGA (sWGA) as indicated in 37 using 20 primers, followed by a WGA. Finally, for the PprfG03 (a P. praefalciparum isolate) and PadlG02 (a $P$. adleri isolate) samples, we used a cell sorting approach38. 


\section{Sample sequencing}

All samples were first sequenced with Illumina. Amplification-free Illumina libraries of 400-600 bp were prepared from the enriched genomic DNA39 and run on MiSeq and HiSeq 2000 (v3 chemistry) Illumina machines.

After the Illumina sequencing, six samples with a combination of the least number of multiple infections (see below) and the lowest level of host contamination were chosen for long read sequencing, using Pacific Biosciences (PacBio). The DNA of the samples (after WGA) was size-selected to $8 \mathrm{~kb}$ and sequenced with the C3/P5 chemistry. The number of SMRT cells (Pacific Bioscience sequencing runs) used varied between samples (Supplementary Table 1).

\section{Genome assembly, genome QC, split of infection \& annotation}

Determination of multiple infections-To initially quantify multiple infections and so allow samples to be selected for PacBio sequencing from those comprising a low number of species, Illumina reads from each sample were mapped against a concatenation of all available $C o x 3$ and $C y t B$ genes of the Laverania from NCBI, using SNP-o-Matic40 (parameter chop=5) to position reads only where they aligned perfectly. SNP-o-Matic returns all the positions of repetitive mapping reads. This output allowed us to count the read depth of these two genes across all species and therefore determine the number and relative amount of different malaria species per sample.

Whole genome amplification (WGA) bias-The uneven coverage that resulted from WGA bias, host contamination and multiple infections presented a challenge for sequence assembly. To overcome the bias and the host contamination, each DNA sample was sequenced deeper than normally necessary. Lower coverage of the subtelomeres was obtained for the sWGA sample (P. blacklocki) meaning that the subtelomeres in that assembly were not as complete as those in the assemblies for other species.

Long reads (Pacific Bioscience) assemblies-Six reference genomes were assembled using HGAP41, with different settings for the genome size parameter, ranging from $23 \mathrm{Mb}$ (P. reichenowi) to $72 \mathrm{Mb}$ (P. billcollinsi). This parameter encodes how many long reads are corrected for use in the assembly and depends on the host contamination and the amount of different isolates in the samples. The obtained contigs from HGAP were ordered with ABACAS42 against a $P$. falciparum 3D7 reference that has no subtelomeric regions. Assembly errors and WGA artefacts were manually corrected using ACT43. After this step, three iterations of ICORN244 were run, followed by another ABACAS step, allowing overlapping contigs to be merged (parameter: ABA_CHECK_OVERLAP=1). For the PrG01, PgabG01 and PadlG01 assembly, we also ran PBjelly to close some of the sequencing gaps 45 .

Host decontamination-To detect and remove sequence data derived from host DNA, contigs were compared with the chimpanzee or gorilla genomes using BLAST. Contigs were considered as host contamination if more than $50 \%$ of their BLAST hits had higher than $95 \%$ identity to any of the great ape genomes. Unordered contigs with a GC content $>32 \%$ 
were searched against the non-redundant nucleotide database, to detect and remove further contaminants.

Resolving multiple infections-The first assembled genome was a single $P$. reichenowi infection, PrG01. We detected low levels of $P$. vivax-like and virus contamination (TT virus, AB038624.1), which were excluded. For quality control, the assembly was compared against the existing PrCDC9 reference genome. The number of Plasmodium interspersed repeats (PIRs) was similar, and there were no breaks in synteny. There were however significantly fewer sequencing gaps and 17 Rep20 regions could be found (a known repeat close to the telomeres in $P$. falciparum). Thus, the assembly of PacBio data (PrG01; Supplementary Table 2) appears to be of higher quality than the existing P. reichenowi PrCDC reference.

The $P$. adleri sample comprised a single infection. Because a large number of cycles of amplification were used, a greater number of SMRT cells were sequenced (Supplementary Table 1) to overcome the problem of uneven coverage resulting in under-represented regions. An estimated genome size of $60 \mathrm{Mb}$ was chosen for the HGAP analysis to ensure that all regions were covered.

PgabG01 was a $P$. gaboni isolate with a $P$. vivax-like co-infection. To detect contigs of $P$. vivax, unordered contigs (those that could not be placed against Pf3D7 using ABACAS) were searched against the protein sequences of $P$. falciparum 3D7 and the $P$. vivax PvP01 reference genome using TBLASTx. For each contig, the relative number of genes hitting against the two genomes was used to assign it to $P$. gaboni or $P$. vivax. In most cases, all genes for a given contig consistently hit only one genome so that the attribution to either species was clear. Overall, $14 \mathrm{Mb}$ of $P$. vivax-like sequences were obtained that will be described elsewhere.

The P. billcollinsi genome (PbilcG01) was obtained from a co-infection with a P. gaboni genome (PgabG02). Rather than ordering the contigs just against Pf3D7 with ABACAS, contigs were ordered against a combined reference comprising P. gaboni (PgabG01) and the Pf3D7 (parameters: overlap $500 \mathrm{bp}$, identity 90\%). The species designation of contigs was confirmed with a TBLASTx searches of annotated genes against a combination of the proteomes of PgabG01 and PrCDC. For subtelomeric gene families, contigs were attributed to species if the hit was significant for one species, not the other. Some of the contigs could not be attributed unambiguously and were discarded. Due to sequencing gaps, some of the core genes are missing from the final assembly.

The sample used to produce the $P$. praefalciparum genome (PprfG01) had a high level of host contamination, a low level of co-infection with $P$. adleri and contained two distinct $P$. praefalciparum genotypes. For the core genome, we used iCORN to select the dominant genotype at each position. Where it was not possible to phase the genotypes, due to a lack of variation, we assumed that they were identical. In the subtelomeres however, it was possible to distinguish but not phase the two P. praefalciparum genotypes resulting in approximately twice the number of vargenes as seen in $P$. falciparum. Due to contamination of construction vectors (E. coli) and host, 29 SMRT cells were sequenced and the HAGP parameter for the assembly size was set to $60 \mathrm{Mb}$. Contigs were screened against $P$. adleri and $P$. falciparum to 
exclude a $P$. adleri co-infection. All of the contigs that had a $P$. falciparum BLAST hit or had no clear hit (such as those containing species-specific gene families) were attributed to the $P$. praefalciparum assembly. Last, all samples (Supplementary Table 1) including five $P$. falciparum genomes were mapped against the Pf3D7, P. praefalciparum and P. adleri assemblies. Contigs were excluded where more normalized hits to the three $P$. adleri samples were found than to one of the two other $P$. praefalciparum samples. Similarly, this method was used to eliminate the remote possibility that any of the contigs in the P. praefalciparum assembly were in fact derived from $P$. falciparum co-infection.

The P. blacklocki sample was from a single infection. Due to sWGA, the PacBio sequence data covered regions not covered by Illumina but due to the bias of the primers, the subtelomeres were not covered fully. However, the internal var gene clusters are all assembled. Some of the core genes from this species are also missing.

Annotation-The genomes were annotated as described in46. In short, the annotation of $P$. falciparum (version July 2015) was transferred with RATT47 and new gene models were called with Augustus48. Obvious structural errors in core genes were manually corrected in Artemis49.

\section{Mapping - generation of further samples}

To generate the gene sequence for different samples, Illumina reads were mapped against a set of reference genomes using BWA50 and default parameters. For the gorilla samples, we mapped against the combined PacBio reference genomes of $P$. adleri, P. blacklocki and $P$. praefalciparum and for the chimpanzee samples, the combined references of $P$. gaboni (PgabG01), P. billcollinsi and P. reichenowi (PrG01). SNPs with Phred score $\geq 100$ were called using GATK UnifiedGenotyper51 v2.0.35 (parameters: -pnrm POOL -ploidy 2 -glm POOLBOTH). From these SNP calls we constructed the new gene set, masking regions in genes with less than 10x coverage of 'properly' (correct distance and orientation) mapped paired reads. To generate the sequences of the other 13 isolates, homozygous SNP calls were obtained (consensus program from bcftools-1.252). We quality controlled the SNP calling by regenerating PrCDC and PgabG02 gene set from PrG01 and PgabG01, respectively and confirmed that they were placed with nearly no differences in a phylogenetic tree.

\section{Orthologous group determination and alignment}

Orthologous groups were identified using OrthoMCL v1.453 across: (i) the seven core Laverania genomes; (ii) the seven core genomes, the Laverania isolates PgabG02, PrCDC and $P$. falciparum IT, as well as two outgroup genomes Plasmodium vivax Sal1 and Plasmodium knowlesi strain H; and (iii) just Pf3D7, PprfG01 and PrG01. P. praefalciparum II was excluded due to its partial genome. From these groups, different complete sets of 1:1 orthologues were extracted:

(1) "Lav12sp" set of 3,369 orthologues across the seven core Laverania species, the PrCDC and $P$. falciparum IT isolates, $P$. vivax and $P$. knowlesi 
(2)
"Lav25st" set of 424 1:1 orthologues from across the 25 Laverania isolates, including the previously published $P$. reichenowi $\mathrm{CDC}$ and five $P$. falciparum isolates (3D7, IT, DD2, HB3 and 7G89).

"Lav7sp" set of 4,350 orthologues from across the seven Laverania reference genomes

"Lav15st" set of 3,808 orthologues, with at least two representative sequences per species, excluding $P$. blacklocki and the most divergent $P$. praefalciparum lineage Pprf3.

"Lav3sp" set of 4,826 1:1 orthologues across all the P. reichenowi, $P$. praefalciparum and $P$. falciparum isolates

The first two sets were used to reconstruct the species tree, the third one for the comparative genomic analyses (introgression, convergence and gene family evolution), the fourth one for the analyses of within species polymorphism and the fifth one for the analysis of $P$. falciparum adaptive evolution.

To reduce the rate of false positives in the evolutionary analyses due to misalignments (e.g. 54), codon-based multiple alignments were performed using PRANK55,56 with the -codon and $+\mathrm{F}$ options, as it was shown to outperform other programs in the context of the detection of positive selection57,58. Prior to aligning codons, low complexity regions were excluded in the nucleotide sequences using dustmasker59 and in amino acid sequences using segmasker60 from NCBI-BLAST. Poorly aligned regions were excluded using Gblocks61, with default settings.

\section{Analysis of interspecific gene flow, introgression or gene transfer}

Species-tree inference-Two ML trees were performed using RAxMLv8.1.2062 to illustrate the phylogenetic relationships between the Laverania species and genotypes studied here using the "Lav12sp" and the "Lav25st" set of orthologues. For each tree, multiple nucleotide alignments of each orthologous group were conducted as described above. Trees were then constructed from the concatenated alignments of the "Lav12sp" set of orthologues for the species tree and the "Lav25st" set for the strain tree using RAxML and the following options "-m GTRGAMMA - $\mathrm{f}$ a -\# 100". Trees were rooted afterwards using $P$. vivax and $P$. knowlesi for the species tree and the $P$. adleri/P. gaboni clade for the genotype tree.

Tree topology test-Interspecific gene flow was investigated by testing congruence between each gene tree topology and the species tree topology. We performed the Shimodaira-Hasegawa test (SH test63) using RAxMLv8.1.20 to test whether the phylogenetic tree for each gene significantly differed from the Laverania species tree. Topology tests were based on multiple nucleotide alignments of the 4,350 "Lav7sp" set of orthologues. For each coding sequence, RAxML was called with the options "-m GTRGAMMA -f h". 


\section{Convergent evolution analyses}

Genome-wide test of convergent evolution-Convergent substitutions can occur by chance and the number of random convergent substitutions between two lineages is correlated with the number of divergent substitutions observed in these two lineages64,65. Excess of convergent substitutions in specific branch pairs can thus be identified by analyzing the correlation between the number of convergent and divergent substitutions between all the branch pairs in a phylogeny using orthogonal regression, and looking for outlier branch pairs: branch pairs with a high positive residual show an excess of convergent substitutions relatively to the number of divergent substitutions64. We used the software Grand-Convergence (available at https://github.com/dekoning-lab/grand-conv) to estimate for each chromosome the numbers of divergent and convergent substitutions between all branch pairs in the Laverania tree and investigate whether branch pairs including Laverania species infecting the same host species (gorilla or chimpanzee) presented an excess of convergence. Analyses were performed under different models of amino-acid evolution: LG, WAG, JONES and DAYHOFF.

\section{Gene-based detection of convergent evolution throughout the Laverania-For} each orthologue of the "Lav7sp" set, the number and percentage of fixed amino acid differences between parasites infecting the same host were calculated, i.e. the number of positions showing the same amino acid within a host species but different amino acid between host species. Alignments of all the available sequences ("Lav15st") from all the sequenced isolates were then used to determine what number of host-specific differences were fixed within each host and each species. To evaluate whether the observed number of host-specific fixed differences in an alignment can be attributed to neutral evolution/ purifying selection alone (with no positive selection), we used a simulation-based approach. For each coding sequence, 1,000 sequences of the same size were simulated, evolving along the same tree with the same specified branch lengths, substitution model, codon frequencies and omega $\left(d_{N} / d_{S}\right)$, using the program Evolver from PAML v4.8a66. The program Codeml from PAML v4.8a66 was first used to estimate the tree, the codon frequencies and the average omega values for each of the coding sequences with fixed amino acid differences. For each simulated dataset, the number of fixed amino-acid differences between the parasites infecting a same host was estimated. The probability of observing $n$ fixed differences was then computed as the proportion of the simulated dataset of 1000 sequences that showed at least the same number of fixed differences as observed in the real data.

\section{Tests for positive selection}

Branch site tests-To search for genes that have been subjected to positive selection in the $P$. falciparum lineage alone after the divergence from $P$. praefalciparum, we used the updated Branch site test 67 implemented in PAML v4.4c 66. This test detects sites that have undergone positive selection in a specific branch of the phylogenetic tree (foreground branch). The "Lav3sp" set of 4,826 orthologuous groups between $P$. reichenowi, $P$. praefalciparum $I$ and $P$. falciparum was used for the test. $d_{\mathrm{N}} / d_{\mathrm{S}}$ ratio estimates per branch and genes were obtained using Codeml (PAML v4.4c) with a free-ratio model of evolution. This identified 139 genes with a significant signal of positive selection in $P$. falciparum only. 
A Branch Site test was also applied, for each gene, on each terminal branch of the entire species tree using the "Lav7sp" dataset. $d_{\mathrm{N}} / d_{\mathrm{S}}$ ratio estimates per branch and genes were obtained using Codeml (PAML v4.4c) with a free-ratio model of evolution, Figure 2.

McDonald-Kreitman (MK) tests-Selection in P. falciparum was also tested using McDonald-Kreitman (MK) tests 68 to compare the polymorphism within species to the divergence between species, using $P$. praefalciparum as the outgroup. Analyses were performed using the 4,826 "Lav3sp" set of orthologues. MK tests were performed as described before9. Thirty-five genes had an MK ratio significantly higher than 1 .

\section{Gene Ontology enrichment analyses}

Analysis of Gene Ontology (GO) term-enrichment was performed in R, using TopGO69 with default parameters. GO annotations from GeneDB were used but with unreviewed automated annotations excluded.

\section{Gene family analyses}

To estimate the differential abundance of gene families across species, the Gene products and the Pfam domains were counted and analysed by the variance of the occurrence. Unless otherwise stated, trees were constructed using PhyML70 (default parameters) or RAxML62 (model estimated) from alignments generated with Muscle71 and trimmed with Gblocks61 in Seaview72 with default values. Many of the findings were confirmed manually through ACT and bamview49. The analysis of the vargenes was performed on vargenes larger than $2.5 \mathrm{~kb}$. Domains were called with the HMMer models from varDom73. Distance matrices were generated based on BLASTp scores, without filtering low complexity regions. Representation was done in $\mathrm{R}$ through the heatmap.2 program from gplot (see also Supplementary Note 3).

Allelic dimorphisms-For the analysis of dimorphism in msp, all sequences available for the Laverania were downloaded from Uniprot74. Data were subsampled to obtain a similar number of sequences for each group. Phylogenetic trees were constructed with PhyML70, using default parameters and drawn in Figtree. The eba-175 alignment was visualized with Jalview75.

\section{Divergence Dating}

Alignments of the Laverania included intergenic regions where possible. Assuming 402-681 mitotic events per year (Supplementary Note 1) and a mutation rate of 3.78E-10 for 4 mitotic events76,77(mutation rate from latter paper was taken from Pf3D7 line without drugs), equivalent to around 0.9-1.5 mutations per genome per year. Although we observed similar mutation rates in clinical samples (Supplementary Note 1), these estimates have potential errors and therefore we report ratios of divergence times in the figures that are robust to errors in these parameters. For coalescence based estimates of speciation times, GPhocs78 was used and multiple sequentially Markovian coalescent (MSMC) on segregating sites 79 was used to estimate the $P$. falciparum bottleneck. 


\section{Supplementary Material}

Refer to Web version on PubMed Central for supplementary material.

\section{Acknowledgments}

This work was funded by ANR ORIGIN JCJC 2012, LMI ZOFAC, CNRS, CIRMF, IRD and the Wellcome Trust (grants WT 098051 and WT 206194 to the Sanger Institute, 104792/Z/14/Z to CN). TC holds a MRC DTP Studentship. We thank Gavin Rutledge for performing the sWGA and Julian Rayner and Francisco J. Ayala for helpful discussion. We thank the PlasmoDB team for promptly making these data available.

\section{References}

1. Prugnolle F, et al. African great apes are natural hosts of multiple related malaria species, including Plasmodium falciparum. Proceedings of the National Academy of Sciences of the United States of America. 2010; 107:1458-1463. DOI: 10.1073/pnas.0914440107 [PubMed: 20133889]

2. Liu W, et al. Origin of the human malaria parasite Plasmodium falciparum in gorillas. Nature. 2010; 467:420-U467. DOI: 10.1038/nature09442 [PubMed: 20864995]

3. Ollomo B, et al. A New Malaria Agent in African Hominids. PLoS Pathog. 2009; 5:e1000446.doi: 10.1371/journal.ppat.1000446 [PubMed: 19478877]

4. Liu W, et al. Multigenomic Delineation of Plasmodium Species of the Laverania Subgenus Infecting Wild-Living Chimpanzees and Gorillas. Genome biology and evolution. 2016; 8:1929-1939. DOI: 10.1093/gbe/evw128 [PubMed: 27289102]

5. Boundenga L, et al. Diversity of malaria parasites in great apes in Gabon. Malaria journal. 2015; 14:111.doi: 10.1186/s12936-015-0622-6 [PubMed: 25889049]

6. Sundararaman SA, et al. Genomes of cryptic chimpanzee Plasmodium species reveal key evolutionary events leading to human malaria. Nature communications. 2016; 7:11078.doi: 10.1038/ ncomms 11078

7. Silva JC, Egan A, Arze C, Spouge JL, Harris DG. A New Method for Estimating Species Age Supports the Coexistence of Malaria Parasites and Their Mammalian Hosts. Molecular biology and evolution. 2015; 32:1354-1364. DOI: 10.1093/molbev/msv005 [PubMed: 25589738]

8. Volkman SK, et al. Recent origin of Plasmodium falciparum from a single progenitor. Science. 2001; 293:482-484. DOI: 10.1126/science.1059878 [PubMed: 11463913]

9. Otto TD, et al. Genome sequencing of chimpanzee malaria parasites reveals possible pathways of adaptation to human hosts. Nature communications. 2014; 5:4754.doi: 10.1038/ncomms5754

10. Larremore DB, et al. Ape parasite origins of human malaria virulence genes. Nature communications. 2015; 6:8368.doi: 10.1038/ncomms9368

11. Pacheco MA, et al. Timing the origin of human malarias: the lemur puzzle. BMC evolutionary biology. 2011; 11:299.doi: 10.1186/1471-2148-11-299 [PubMed: 21992100]

12. Behar DM, et al. The dawn of human matrilineal diversity. American journal of human genetics. 2008; 82:1130-1140. DOI: 10.1016/j.ajhg.2008.04.002 [PubMed: 18439549]

13. Palstra FP, Fraser DJ. Effective/census population size ratio estimation: a compendium and appraisal. Ecology and evolution. 2012; 2:2357-2365. DOI: 10.1002/ece3.329 [PubMed: 23139893]

14. Roy SW. The Plasmodium gaboni genome illuminates allelic dimorphism of immunologically important surface antigens in P. falciparum. Infection, Genetics and Evolution. 2015; 36:441-449. DOI: 10.1016/j.meegid.2015.08.014

15. Tanabe K, Mackay M, Goman M, Scaife JG. Allelic dimorphism in a surface antigen gene of the malaria parasite Plasmodium falciparum. Journal of molecular biology. 1987; 195:273-287. DOI: 10.1016/0022-2836(87)90649-8 [PubMed: 3079521]

16. Yasukochi Y, Naka I, Patarapotikul J, Hananantachai H, Ohashi J. Genetic evidence for contribution of human dispersal to the genetic diversity of EBA-175 in Plasmodium falciparum. Malar J. 2015; 14:293.doi: 10.1186/s12936-015-0820-2 [PubMed: 26231699] 
17. Malaria Genomic Epidemiology, N. Band G, Rockett KA, Spencer CC, Kwiatkowski DP. A novel locus of resistance to severe malaria in a region of ancient balancing selection. Nature. 2015; 526:253-257. DOI: 10.1038/nature15390 [PubMed: 26416757]

18. Makanga B, et al. Ape malaria transmission and potential for ape-to-human transfers in Africa. Proceedings of the National Academy of Sciences of the United States of America. 2016; 1603008113. doi: 10.1073/pnas.1603008113

19. Wanaguru M, Liu W, Hahn BH, Rayner JC, Wright GJ. RH5-Basigin interaction plays a major role in the host tropism of Plasmodium falciparum. Proceedings of the National Academy of Sciences. 2013; 110:20735-20740. DOI: 10.1073/pnas.1320771110

20. Wright KE, et al. Structure of malaria invasion protein RH5 with erythrocyte basigin and blocking antibodies. Nature. 2014; 515:427.doi: 10.1038/nature13715 [PubMed: 25132548]

21. Triglia T, Thompson JK, Cowman AF. An EBA175 homologue which is transcribed but not translated in erythrocytic stages of Plasmodium falciparum. Mol Biochem Parasitol. 2001; 116:5563. DOI: 10.1016/S0166-6851(01)00303-6 [PubMed: 11463466]

22. Farrell A, et al. A DOC2 Protein Identified by Mutational Profiling Is Essential for Apicomplexan Parasite Exocytosis. Science. 2012; 335:218-221. DOI: 10.1126/science.1210829 [PubMed: 22246776]

23. Ramiro RS, et al. Hybridization and pre-zygotic reproductive barriers in Plasmodium. Proceedings of the Royal Society B-Biological Sciences. 2015; 282doi: 10.1098/rspb.2014.3027

24. Eksi S, et al. Malaria transmission-blocking antigen, Pfs230, mediates human red blood cell binding to exflagellating male parasites and oocyst production. Molecular Microbiology. 2006; 61:991-998. DOI: 10.1111/j.1365-2958.2006.0528.x [PubMed: 16879650]

25. Mundwiler-Pachlatko E, Beck HP. Maurer's clefts, the enigma of Plasmodium falciparum. Proceedings of the National Academy of Sciences of the United States of America. 2013; 110:19987-19994. DOI: 10.1073/pnas.1309247110 [PubMed: 24284172]

26. Bethke LL, et al. Duplication, gene conversion, and genetic diversity in the species-specific acylCoA synthetase gene family of Plasmodium falciparum. Mol Biochem Parasitol. 2006; 150:10-24. DOI: 10.1016/j.molbiopara.2006.06.004 [PubMed: 16860410]

27. Cunningham D, Lawton J, Jarra W, Preiser P, Langhorne J. The pir multigene family of Plasmodium: antigenic variation and beyond. Mol Biochem Parasitol. 2010; 170:65-73. DOI: 10.1016/j.molbiopara.2009.12.010 [PubMed: 20045030]

28. Niang M, et al. STEVOR is a Plasmodium falciparum erythrocyte binding protein that mediates merozoite invasion and rosetting. Cell Host Microbe. 2014; 16:81-93. DOI: 10.1016/j.chom. 2014.06.004 [PubMed: 25011110]

29. Kraemer SM, Smith JD. A family affair: var genes, PfEMP1 binding, and malaria disease. Curr Opin Microbiol. 2006; 9:374-380. DOI: 10.1016/j.mib.2006.06.006 [PubMed: 16814594]

30. Gardner MJ, et al. Genome sequence of the human malaria parasite Plasmodium falciparum. Nature. 2002; 419:498-511. DOI: 10.1038/nature01097 [PubMed: 12368864]

31. Bordbar B, et al. Genetic diversity of VAR2CSA ID1-DBL2Xb in worldwide Plasmodium falciparum populations: impact on vaccine design for placental malaria. Infection, genetics and evolution : journal of molecular epidemiology and evolutionary genetics in infectious diseases. 2014; 25:81-92. DOI: 10.1016/j.meegid.2014.04.010

32. Frank M, Dzikowski R, Amulic B, Deitsch K. Variable switching rates of malaria virulence genes are associated with chromosomal position. Mol Microbiol. 2007; 64:1486-1498. DOI: 10.1111/j. 1365-2958.2007.05736.x [PubMed: 17555435]

33. Scally A, Durbin R. Revising the human mutation rate: implications for understanding human evolution. Nature reviews. Genetics. 2012; 13:745-753. DOI: 10.1038/nrg3295

34. Carter R, Mendis KN. Evolutionary and historical aspects of the burden of malaria. Clinical microbiology reviews. 2002; 15:564-594. DOI: 10.1128/CMR.15.4.564-594.2002 [PubMed: 12364370]

35. Auburn S, et al. An effective method to purify plasmodium falciparum dna directly from clinical blood samples for whole genome high-throughput sequencing. PLoS ONE. 2011; 6doi: 10.1371/ journal.pone. 0022213 
36. Oyola SO, et al. Optimized whole-genome amplification strategy for extremely AT-biased template. DNA research : an international journal for rapid publication of reports on genes and genomes. 2014; 21:661-671. DOI: 10.1093/dnares/dsu028 [PubMed: 25240466]

37. Oyola SO, et al. Whole genome sequencing of Plasmodium falciparum from dried blood spots using selective whole genome amplification. bioRxiv. 2016; doi: 10.1101/067546

38. Boissiere A, et al. Isolation of Plasmodium falciparum by flow-cytometry: implications for singletrophozoite genotyping and parasite DNA purification for whole-genome high-throughput sequencing of archival samples. Malaria Journal. 2012; 11:163.doi: 10.1186/1475-2875-11-163 [PubMed: 22583664]

39. Quail MA, et al. Optimal enzymes for amplifying sequencing libraries. Nat Methods. 2012; 9:1011. DOI: $10.1038 /$ nmeth. 1814

40. Manske H, Kwiatkowski D. SNP-o-matic. Bioinformatics. 2009; 25:2434-2435. DOI: 10.1093/ bioinformatics/btp403 [PubMed: 19574284]

41. Chin CS, et al. Nonhybrid, finished microbial genome assemblies from long-read SMRT sequencing data. Nat Methods. 2013; 10:563-569. DOI: 10.1038/nmeth.2474 [PubMed: 23644548]

42. Assefa S, Keane TM, Otto TD, Newbold C, Berriman M. ABACAS: algorithm-based automatic contiguation of assembled sequences. Bioinformatics. 2009; 25(15):1968-1969. [PubMed: 19497936]

43. Carver T, et al. Artemis and ACT: viewing, annotation and comparing sequences stored in relational database. Bioinformatics. 2008; 24:2672-2676. DOI: 10.1093/bioinformatics/btn529 [PubMed: 18845581]

44. Otto TD, Sanders M, Berriman M, Newbold C. Iterative Correction of Reference Nucleotides (iCORN) using second generation sequencing technology. Bioinformatics. 2010; 26:1704-1707. DOI: 10.1093/bioinformatics/btq269 [PubMed: 20562415]

45. English AC, et al. Mind the gap: upgrading genomes with Pacific Biosciences RS long-read sequencing technology. PLoS One. 2012; 7:e47768.doi: 10.1371/journal.pone.0047768 [PubMed: 23185243]

46. Otto TD. From sequence mapping to genome assemblies. Methods Mol Biol. 2015; 1201:19-50. DOI: 10.1007/978-1-4939-1438-8_2 [PubMed: 25388106]

47. Otto TD, Dillon GP, Degrave WS, Berriman M. RATT: Rapid Annotation Transfer Tool. Nucleic Acids Research. 2011; 39:e57.doi: 10.1093/nar/gkq1268 [PubMed: 21306991]

48. Stanke M, et al. AUGUSTUS: ab initio prediction of alternative transcripts. Nucleic Acids Research. 2006; 34:W435-439. DOI: 10.1093/nar/gk1200 [PubMed: 16845043]

49. Carver T, et al. BamView: visualizing and interpretation of next-generation sequencing read. Briefings in bioinformatics. 2013; doi: 10.1093/bib/bbr073

50. Li H, Durbin R. Fast and accurate short read alignment with Burrows-Wheeler transform. Bioinformatics. 2009; 25:1754-1760. DOI: 10.1093/bioinformatics/btp324 [PubMed: 19451168]

51. McKenna A, et al. The Genome Analysis Toolkit: a MapReduce framework for analyzing nextgeneration DNA sequencing data. Genome Res. 2010; 20:1297-1303. DOI: 10.1101/gr. 107524.110 [PubMed: 20644199]

52. Danecek P, et al. The variant call format and VCFtools. Bioinformatics. 2011; 27:2156-2158. DOI: 10.1093/bioinformatics/btr330 [PubMed: 21653522]

53. Li L, Stoeckert CJ Jr, Roos DS. OrthoMCL: identification of ortholog groups for eukaryotic genomes. Genome Res. 2003; 13:2178-2189. DOI: 10.1101/gr.1224503 [PubMed: 12952885]

54. Jordan G, Goldman N. The Effects of Alignment Error and Alignment Filtering on the Sitewise Detection of Positive Selection. Molecular biology and evolution. 2012; 29:1125-1139. DOI: 10.1093/molbev/msr272 [PubMed: 22049066]

55. Loytynoja A, Goldman N. An algorithm for progressive multiple alignment of sequences with insertions. Proceedings of the National Academy of Sciences of the United States of America. 2005; 102:10557-10562. DOI: 10.1073/pnas.0409137102 [PubMed: 16000407]

56. Loytynoja A, Goldman N. Phylogeny-aware gap placement prevents errors in sequence alignment and evolutionary analysis. Science. 2008; 320:1632-1635. DOI: 10.1126/science.1158395

[PubMed: 18566285] 
57. Fletcher W, Yang Z. The Effect of Insertions, Deletions, and Alignment Errors on the Branch-Site Test of Positive Selection. Molecular biology and evolution. 2010; 27:2257-2267. DOI: 10.1093/ molbev/msq115 [PubMed: 20447933]

58. Markova-Raina P, Petrov D. High sensitivity to aligner and high rate of false positives in the estimates of positive selection in the 12 Drosophila genomes. Genome Research. 2011; 21:863874. DOI: 10.1101/gr.115949.110 [PubMed: 21393387]

59. Morgulis A, Gertz EM, Schäffer AA, Agarwala R. A Fast and Symmetric DUST Implementation to Mask Low-Complexity DNA Sequences. Journal of Computational Biology. 2006; 13:10281040. DOI: 10.1089/cmb.2006.13.1028 [PubMed: 16796549]

60. Wootton JC, Federhen S. Statistics of local complexity in amino acid sequences and sequence databases. Computers \& Chemistry. 1993; 17:149-163. DOI: 10.1016/0097-8485(93)85006-X

61. Castresana J. Selection of Conserved Blocks from Multiple Alignments for Their Use in Phylogenetic Analysis. Molecular biology and evolution. 2000; 17:540-552. DOI: 10.1093/ oxfordjournals.molbev.a026334 [PubMed: 10742046]

62. Stamatakis A. RAxML version 8: a tool for phylogenetic analysis and post-analysis of large phylogenies. Bioinformatics. 2014; 30:1312-1313. DOI: 10.1093/bioinformatics/btu033 [PubMed: 24451623]

63. Shimodaira H, Hasegawa M. Multiple Comparisons of Log-Likelihoods with Applications to Phylogenetic Inference. Molecular biology and evolution. 1999; 16:1114.doi: 10.1093/ oxfordjournals.molbev.a026201

64. Castoe TA, et al. Evidence for an ancient adaptive episode of convergent molecular evolution. Proceedings of the National Academy of Sciences of the United States of America. 2009; 106:8986-8991. DOI: 10.1073/pnas.0900233106 [PubMed: 19416880]

65. Thomas GWC, Hahn MW. Determining the Null Model for Detecting Adaptive Convergence from Genomic Data: A Case Study using Echolocating Mammals. Molecular biology and evolution. 2015; 32:1232-1236. DOI: 10.1093/molbev/msv013 [PubMed: 25631926]

66. Yang Z. PAML 4: Phylogenetic Analysis by Maximum Likelihood. Molecular biology and evolution. 2007; 24:1586-1591. DOI: 10.1093/molbev/msm088 [PubMed: 17483113]

67. Zhang J, Nielsen R, Yang Z. Evaluation of an Improved Branch-Site Likelihood Method for Detecting Positive Selection at the Molecular Level. Molecular biology and evolution. 2005; 22:2472-2479. DOI: 10.1093/molbev/msi237 [PubMed: 16107592]

68. McDonald JH, Kreitman M. Adaptive protein evolution at the Adh locus in Drosophila. Nature. 1991; 351:652-654. DOI: 10.1038/351652a0 [PubMed: 1904993]

69. Rahnenfuhrer, AAaJ. topGO: Enrichment analysis for Gene Ontology. R package. 2010; doi: 10.18129/B9.bioc.topGO

70. Guindon S, et al. New algorithms and methods to estimate maximum-likelihood phylogenies: assessing the performance of PhyML 3.0. Syst Biol. 2010; 59:307-321. DOI: 10.1093/sysbio/ syq010 [PubMed: 20525638]

71. Edgar RC. MUSCLE: multiple sequence alignment with high accuracy and high throughput. Nucleic Acids Res. 2004; 32:1792-1797. DOI: 10.1093/nar/gkh340 [PubMed: 15034147]

72. Gouy M, Guindon S, Gascuel O. SeaView version 4: A multiplatform graphical user interface for sequence alignment and phylogenetic tree building. Molecular biology and evolution. 2010; 27:221-224. DOI: 10.1093/molbev/msp259 [PubMed: 19854763]

73. Rask TS, Hansen DA, Theander TG, Gorm Pedersen A, Lavstsen T. Plasmodium falciparum erythrocyte membrane protein 1 diversity in seven genomes--divide and conquer. PLoS Comput Biol. 2010; 6doi: 10.1371/journal.pcbi.1000933

74. UniProt, C. UniProt: a hub for protein information. Nucleic Acids Res. 2015; 43:D204-212. DOI: 10.1093/nar/gku989 [PubMed: 25348405]

75. Waterhouse AM, Procter JB, Martin DM, Clamp M, Barton GJ. Jalview Version 2--a multiple sequence alignment editor and analysis workbench. Bioinformatics. 2009; 25:1189-1191. DOI: 10.1093/bioinformatics/btp033 [PubMed: 19151095]

76. Claessens A, et al. Generation of antigenic diversity in Plasmodium falciparum by structured rearrangement of Var genes during mitosis. PLoS Genet. 2014; 10:e1004812.doi: 10.1371/ journal.pgen.1004812 [PubMed: 25521112] 
77. Bopp SE, et al. Mitotic evolution of Plasmodium falciparum shows a stable core genome but recombination in antigen families. PLoS Genet. 2013; 9:e1003293.doi: 10.1371/journal.pgen. 1003293 [PubMed: 23408914]

78. Gronau I, Hubisz MJ, Gulko B, Danko CG, Siepel A. Bayesian inference of ancient human demography from individual genome sequences. Nat Genet. 2011; 43:1031-1034. DOI: 10.1038/ng.937 [PubMed: 21926973]

79. Schiffels S, Durbin R. Inferring human population size and separation history from multiple genome sequences. Nat Genet. 2014; 46:919-925. DOI: 10.1038/ng.3015 [PubMed: 24952747] 
a

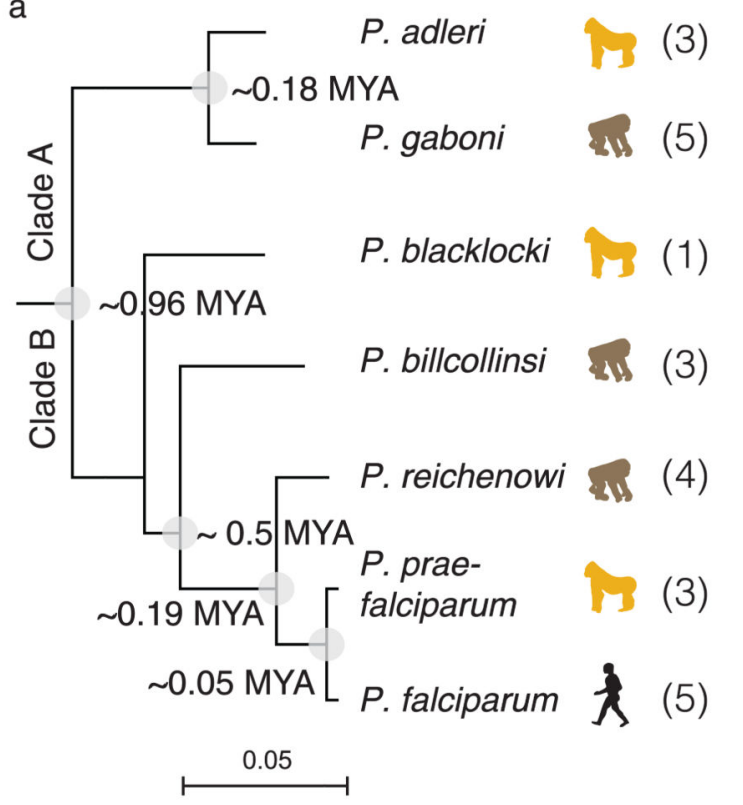

b

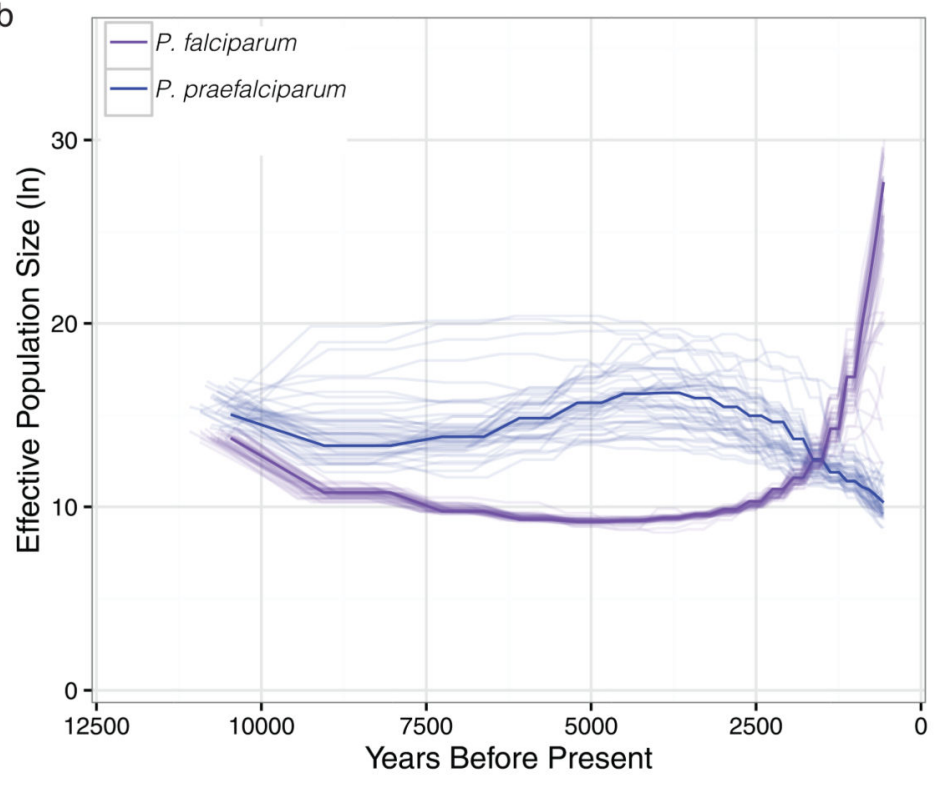

Figure 1. Overview of the dating of the evolution of the Laverania.

(a) Maximum likelihood tree of the Laverania based on the "Lav12sp" set of orthologues.

All bootstrap values are 100. Coalescence based estimates of the timing of speciation events are displayed on nodes (MYA - million years ago), based on intergenic and genic alignments. (b) Multiple sequentially Markovian coalescent estimates of the effective population size $(\mathrm{Ne})$ in the $P$. falciparum and $P$. praefalciparum population. Assuming our estimate of the number of mitotic events per year, a bottleneck occurred in P. falciparum 4,000-6,000 years ago. The y-axis shows the natural logarithm (Ln) of Ne. Bootstrapping (pale lines) was performed by randomly resampling segregating sites from the input 50 times. 


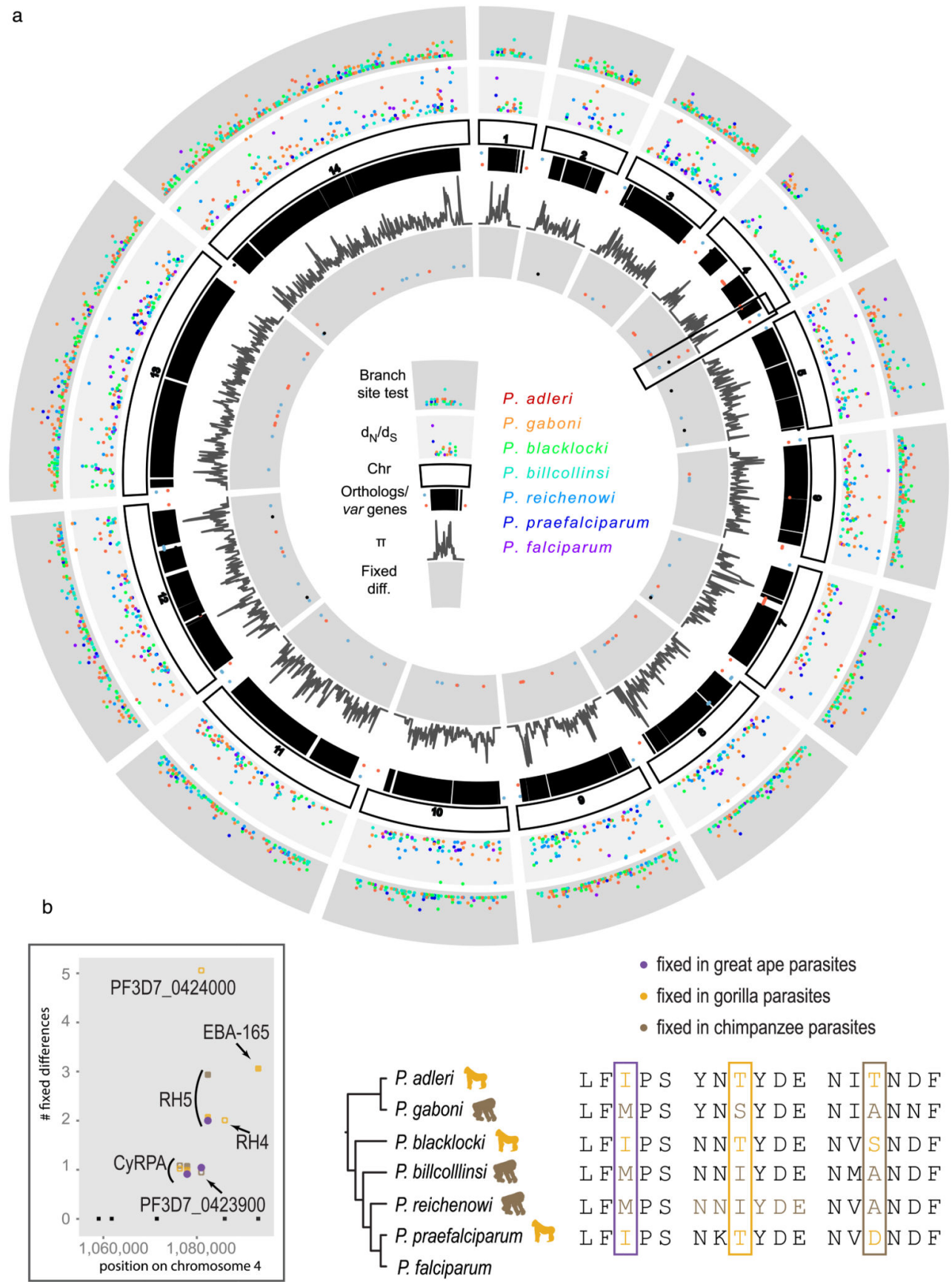

Figure 2. Overview of the analyses of core genes over all Laverania genomes.

(a) Summary of evolution of core genes. From outer to inner track: scatterplot of branch site test for each genome (see Supplementary Table 4 for $P$. falciparum data); per-species $d_{\mathrm{N}} / d_{\mathrm{S}}$ values $\left(0.5<d_{\mathrm{N}} / d_{\mathrm{S}}<2\right)$; orthologues represented by vertical black lines under the chromosome track represent, with dots representing $P$. falciparum 3D7 vargenes on the forward (blue) or reverse strands (red), or var pseudogenes (black); average of the relative polymorphism $(\pi)$ across species, with the underlying $\pi$ for each species calculated from multiple strains ("Lav15st" dataset) and normalized by the average for that species; 
signatures of convergent evolution based on host-specific fixed differences analysis with the chromosome 4 region that includes the Rh5 locus highlighted (black box). (b) Magnified view of the $R h 5$ region that is enriched with host specific fixed differences. Convergent evolution analysis was performed using orthologues conserved across the Laverania. Filled circles represent the subset of differences that were fixed within all the isolates available ("Lav15st" set) and for which we could reject neutral evolution (for the gene list see Supplementary Table 5). 


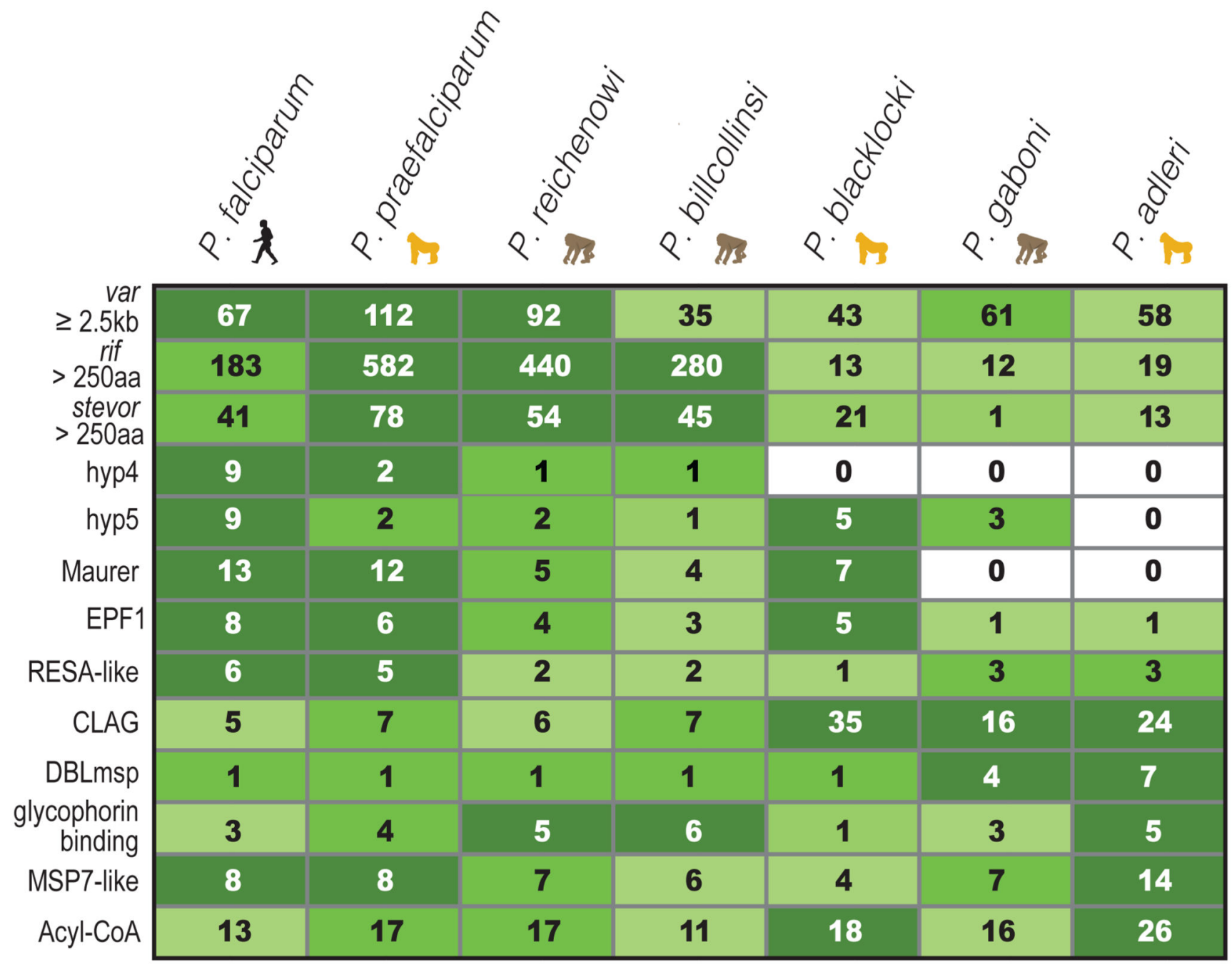

Figure 3. Gene families in the Laverania.

Distribution of major multigene families including var and those that show significant copy number variation among lineages. Data from $P$. praefalciparum include the subtelomeric gene families from the two infecting genotypes. Assembly of $P$. billcollinsi is incomplete in the subtelomeres. 


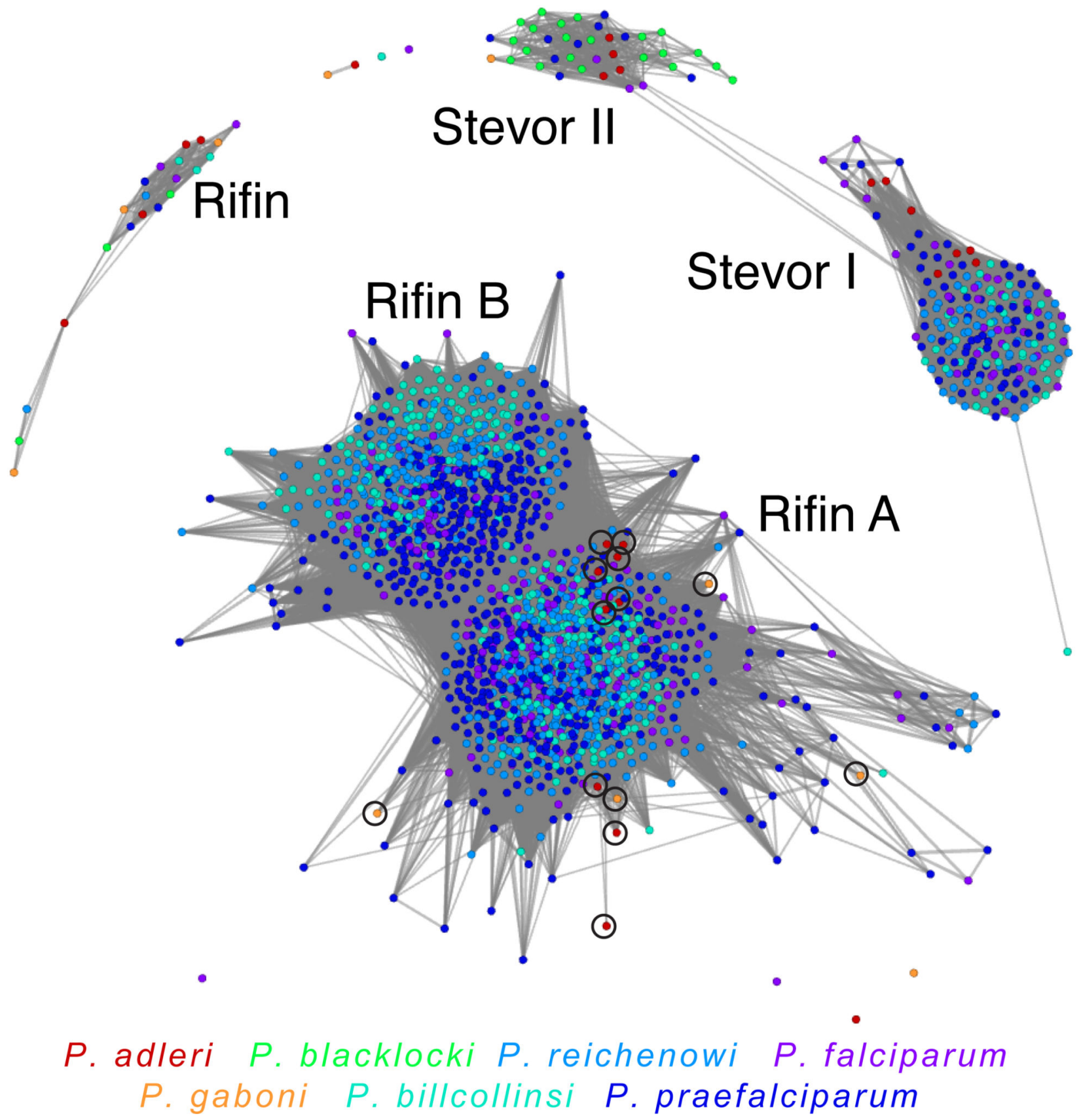

Figure 4. Clustering of Pir (Rifin and Stevor) proteins families.

Graphical representation of similarity between all pir proteins $>250 \mathrm{aa}$, coloured by species. A BLAST cut-off of $45 \%$ global identity was used (see methods). More connected genes are more similar. Black circles highlight Clade A rifin proteins that cluster with Clade B rifin proteins. 


\begin{tabular}{|c|c|c|c|c|c|c|c|c|c|c|c|c|c|c|c|c|c|}
\hline a & CIDRa & CIDRb & CIDRd & CIDRg & CIDRn & CIDRpam & DBLpam1 & DBLpam2 & DBLpam3 & DBLa & DBLb & DBLd & DBLe & DBLg & DBLz & Duffy & ATS \\
\hline P. adleri & 1 & 1 & 0 & 0 & 14 & 4 & 1 & 15 & 9 & 2 & 63 & 0 & 106 & 67 & 20 & 77 & 39 \\
\hline P. gaboni & 1 & 1 & 0 & 0 & 8 & 16 & 3 & 30 & 20 & 2 & 47 & 0 & 84 & 43 & 16 & 48 & 41 \\
\hline P. blacklocki & 0 & 1 & 0 & 0 & 0 & 0 & 0 & 0 & 0 & 1 & 17 & 0 & 16 & 55 & 7 & 0 & 34 \\
\hline P. billcollinsi & 31 & 28 & 0 & 5 & 0 & 0 & 1 & 0 & 0 & 30 & 9 & 34 & 4 & 2 & 1 & 0 & 28 \\
\hline P. reichenowi & 86 & 61 & 1 & 27 & 0 & 1 & 2 & 1 & 1 & 90 & 50 & 85 & 18 & 43 & 8 & 0 & 85 \\
\hline P. praefalciparum & 85 & 48 & 5 & 30 & 0 & 5 & 5 & 5 & 5 & 94 & 86 & 72 & 97 & 84 & 34 & 0 & 105 \\
\hline P. falciparum & 56 & 37 & 2 & 17 & 0 & 1 & 3 & 1 & 1 & 59 & 18 & 53 & 15 & 16 & 7 & 0 & 65 \\
\hline
\end{tabular}

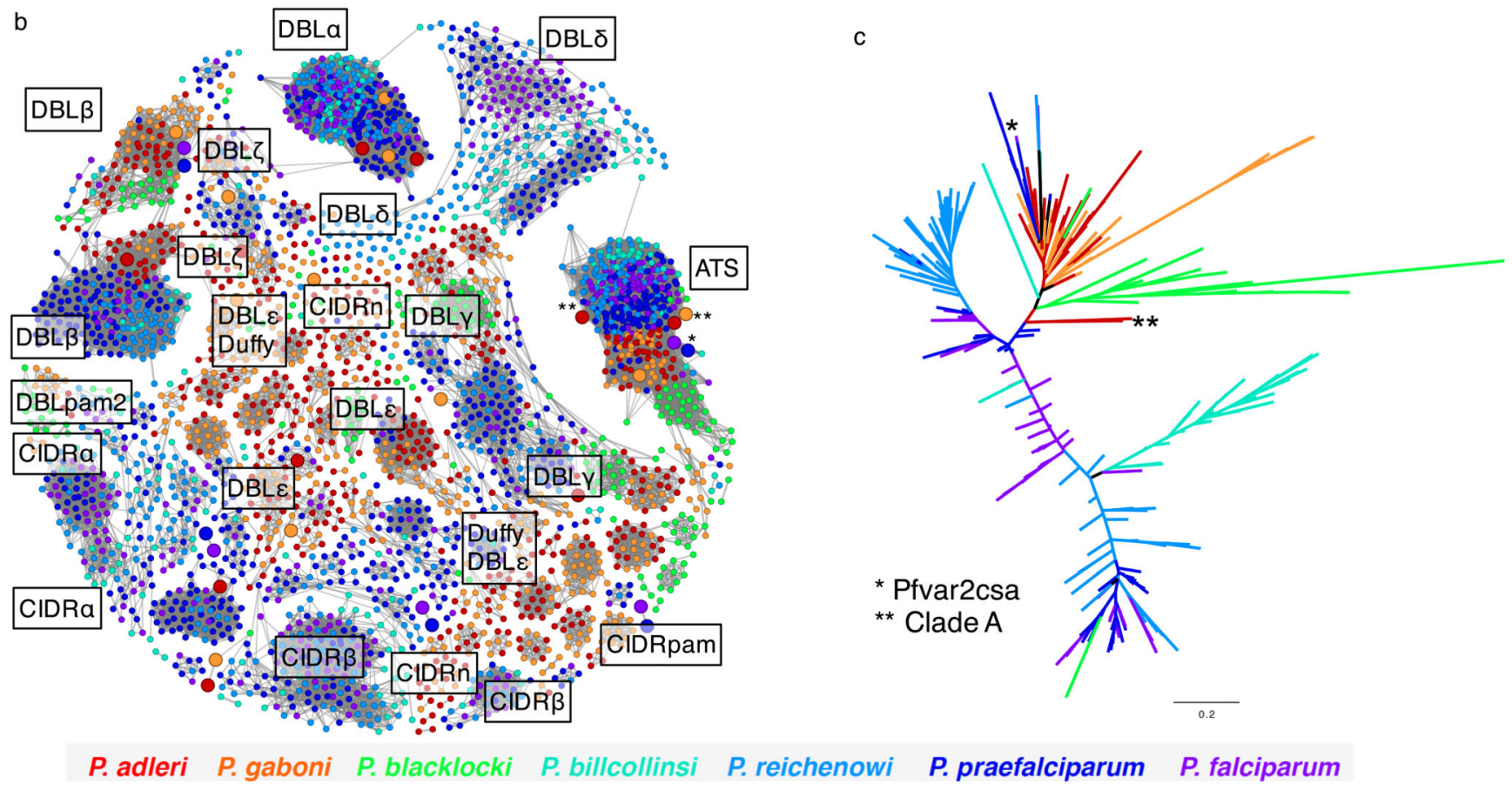

Figure 5. Evolution of var gene domains in the Laverania

(a) Heatmap of numbers of var gene domains in each Laverania species. Duffy represents regions closest to the Pfam Duffy binding domain. CIDRn is a new domain discovered in this study in Clade A. Only domains from vargenes longer than $2.5 \mathrm{~kb}$ were considered. Heat map colours blue-yellow-white indicate decreasing copy numbers. (b) Graphical representation of similarity between domains, using domains from var genes longer than $2.5 \mathrm{~kb}$. Domains are coloured by species and clustered by a minimum BLAST cut-off of $45 \%$ global identity. Larger circles denote vargenes in the opposite orientation. (c) Maximum likelihood trees of the Acidic Terminal Sequence (ATS). Apparent ATS sequences from clade $\mathrm{A}$ that cluster with clade $\mathrm{B}$ are indicated $(* *)$. 


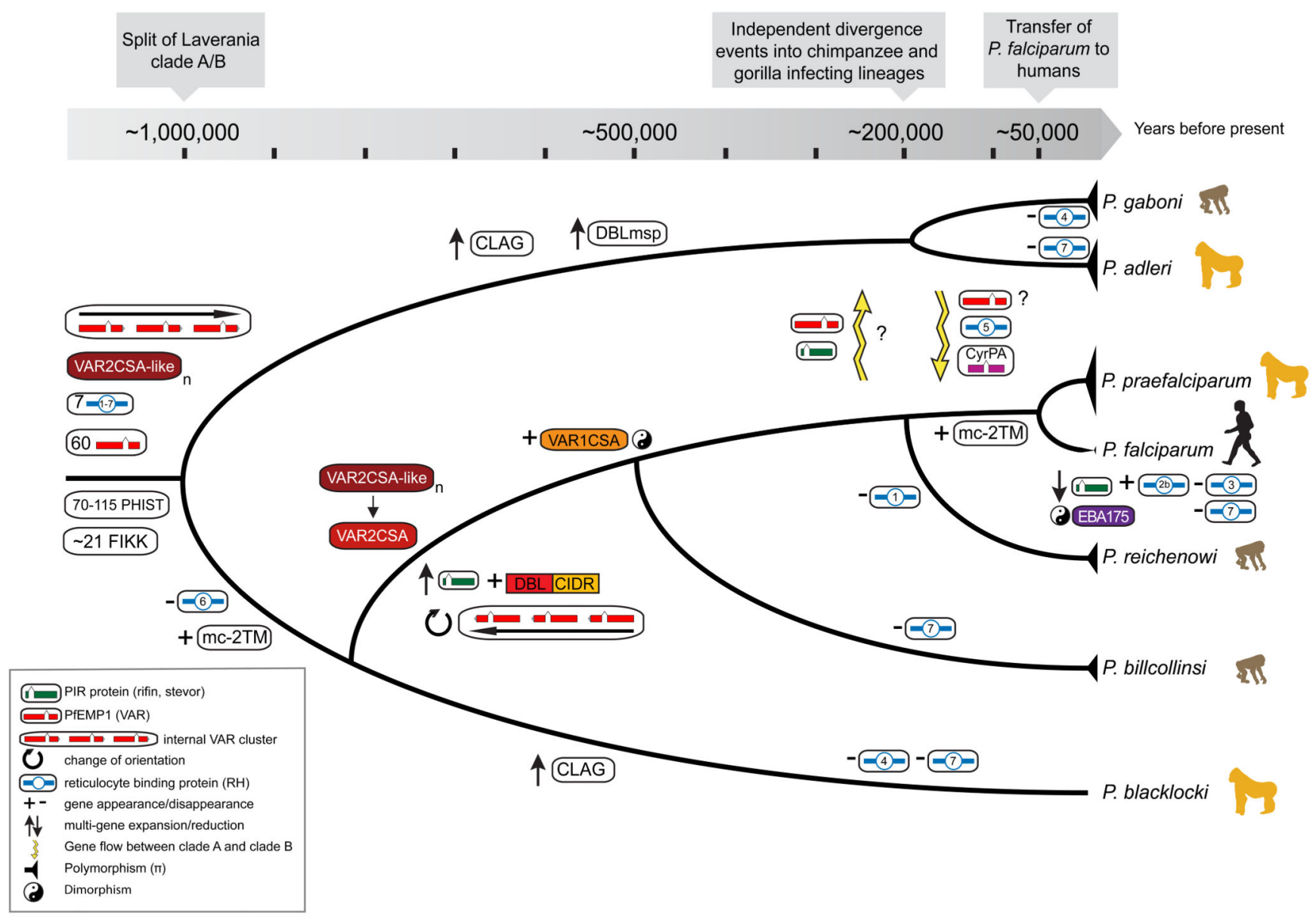

Figure 6. Overview of the genomic evolution of the Laverania subgenus.

The values of polymorphism $(\pi)$ within the species are indicated by triangles of different size at the end of the tree branches, as well the bottleneck in $P$. falciparum (constricted branch width), 5,000 years ago. Also shown are the gene transfers that occurred between certain Clade A and B species and the huge genomic differences that accumulated in Clade B after the divergence with $P$. blacklocki. 